\title{
A Study of Redistribution Phenomenon of Solute Atoms in CoCrTa and CoCr Perpendicular Recording Films by Annealing
}

\author{
Chung Ho HWANG ${ }^{1}$, Taek Dong LEE ${ }^{2}$, Kyung Ho SHIN ${ }^{3}$, and Yong Soo PARK ${ }^{1}$ \\ 1)Dept. of Metal. Eng. Yonsei Univ.,Seodaemun-ku, Seoul 120-749, Korea \\ 2) Dept. of Advanced Materials Eng., KAIST, Dongdaemun-ku, Seoul, 130-650, Korea \\ 3) Division of Metals, KIST, Seongbuk-ku, Seoul 136-791, Korea
}

\begin{abstract}
In the previous work ${ }^{1}$, we have reported Ta addition is effective in increasing perpendicular coercivity of the films with $\mathrm{Cr}$ content lower than 17 at\% $\mathrm{Cr}$ region and not effective in those with higher than $19 \mathrm{at} \% \mathrm{Cr}$ region. Also the higher coercivity of the CoCrTa films is interpreted as the enhanced $\mathrm{Cr}$ segregation than Ta segregation itself. In the present work, we studied the redistributing behaviour of solutes of the as-deposited films by analysing changes of structures and magnetic properties before and after vacuum annealing. It was found that the redistributing atomic species during the annealing is $\mathrm{Cr}$ rather than $\mathrm{Ta}$, which is in good agreement with our previous claims that $\mathrm{Ta}$ enhances $\mathrm{Cr}$ segregation in CoCrTa system.
\end{abstract}

\section{INTRODUCTION}

Higher coercivity at thinner film thickness is an essential requirement for a high density recording medium in perpendicular recording as well as in longitudinal recording. So far $\mathrm{CoCr}$ and $\mathrm{CoCrTa}$ films have been widely studied as strong candidates for the media. CoCrTa perpendicular recording films have superior magnetic properties to $\mathrm{CoCr}$ films. Small addition of $\mathrm{Ta}$ is more effective in raising the perpendicular coercivity than further addition of $\mathrm{Cr}$ to CoCr films containing 19 at $\%$ or less 1 .

We have reported the higher coercivity behaviour of $\mathrm{CoCrTa}$ films is not associated with the segregation of Ta itself but associated with promotion of enhanced segregation of $\mathrm{Cr}$ due to the existence of $\mathrm{Ta}^{1}$. This conclusion was drawn based on grain size examination, $\mathrm{X}$-ray analysis and comparison of magnetic properties. An sputtering result has been reported by other researchers $^{2}$ suggesting Ta may not preferentially segregated but uniformly dispersed within grains in longitudinal sputtered films.

The purpose of this work is to study redistribution behaviour in sputter deposited films of $\mathrm{Co}_{81.4} \mathrm{Cr}_{16.6} \mathrm{Ta}_{2}$ by analysing the changes of magnetic properties and structures by annealing. ${ }_{0}{ }_{81} \mathrm{Cr}_{19}$ films were selected and studied as a reference. By comparing the magnetic properties of CoCrTa films with those of $\mathrm{CoCr}$ films before and after annealing, we tried to analyse the redistributing atomic species.

\section{EXPerimental Procedure}

The films of different compositions were prepared by an rf sputtering method on polyimide substrates. For compositional variation $\mathrm{Ta}$ chips and $\mathrm{Cr}$ chips of $99.99 \%$ purity were placed on $\mathrm{Co}_{83} \mathrm{Cr}_{17}$ and $\mathrm{C}_{81} \mathrm{Cr}_{19}$ alloy targets. The compositions of the films were analysed with EPMA. For annealing experiments, 2.5 $\mu \mathrm{m}$ thick free standing films were prepared by dissolving the substrates and were heat-treated isothermally at various temperatures in a vacuum of better than $1 \times 10^{-5}$ torr. Magnetic properties were measured by a VSM and a torque magnetometer at room temperature. The structures of the films were studied by TEM and XRD.

\section{RESUlts AND DISCUSSION}

Fig. 1 shows the variation of $\mathrm{Ms}$ in $\mathrm{Co}_{81} \mathrm{Cr}_{19}$ and $\mathrm{Co}_{81.4} \mathrm{Cr}_{16.6} \mathrm{Ta}_{2}$ films deposited on $100^{\circ} \mathrm{C}$ substrates with varying annealing temperatures. The change of the

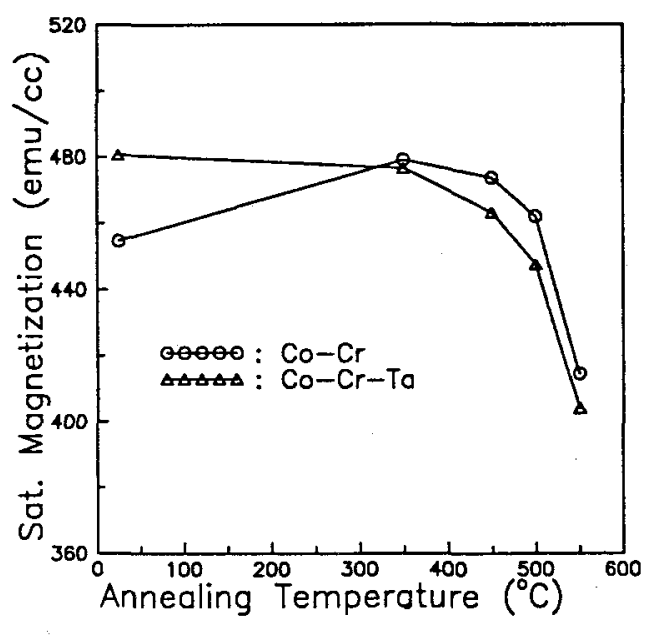

Fig.1 Variation of $\mathrm{Ms}$ of $\mathrm{Co}_{81} \mathrm{Cr}_{19}$ and $\mathrm{Co}_{81.4} \mathrm{Cr}_{16.6} \mathrm{Ta}_{2}$ films with annealing temperature 


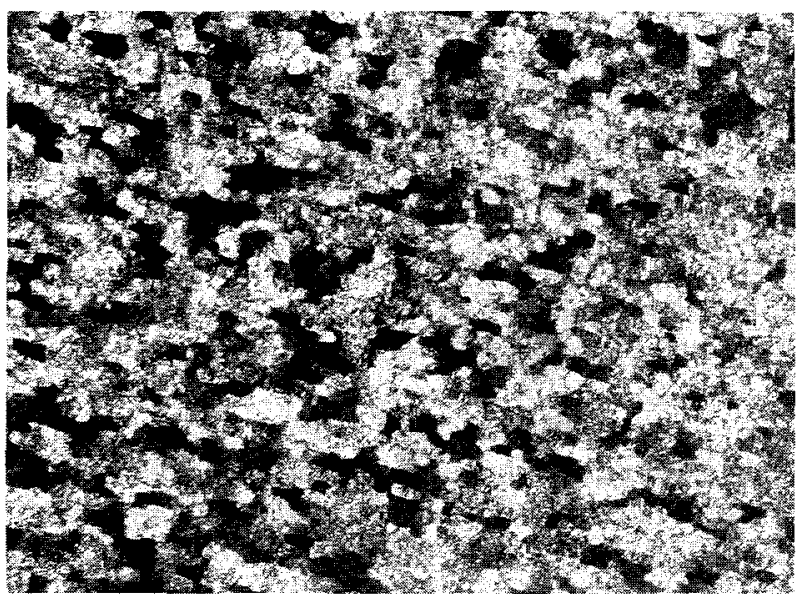

As-sputtered

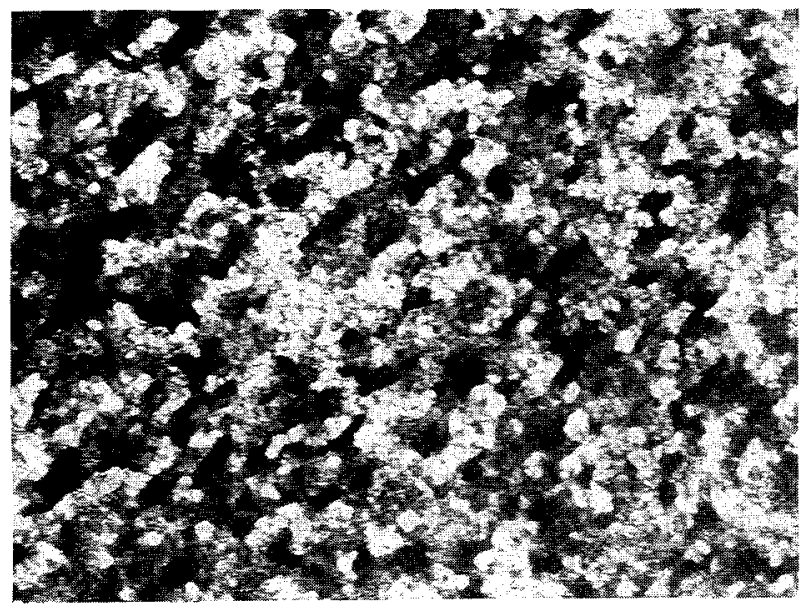

$550^{\circ} \mathrm{C}$

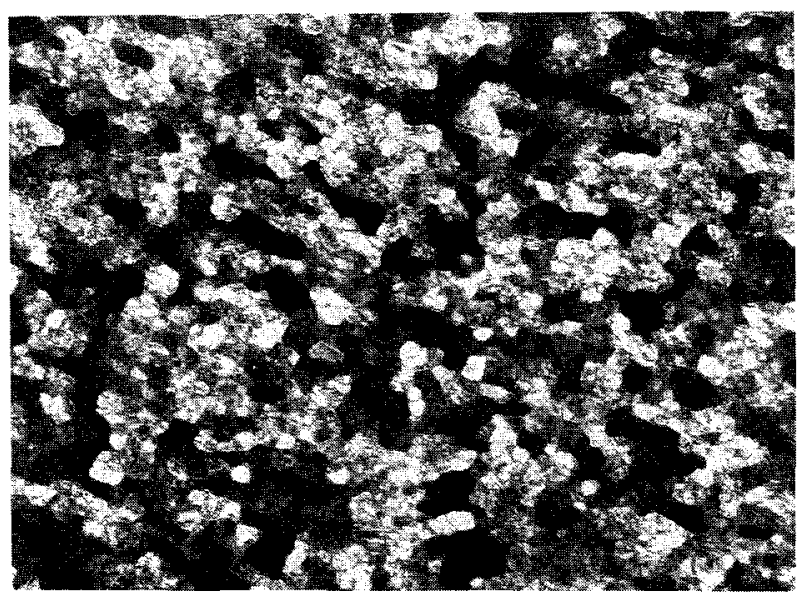

$650^{\circ} \mathrm{C}$

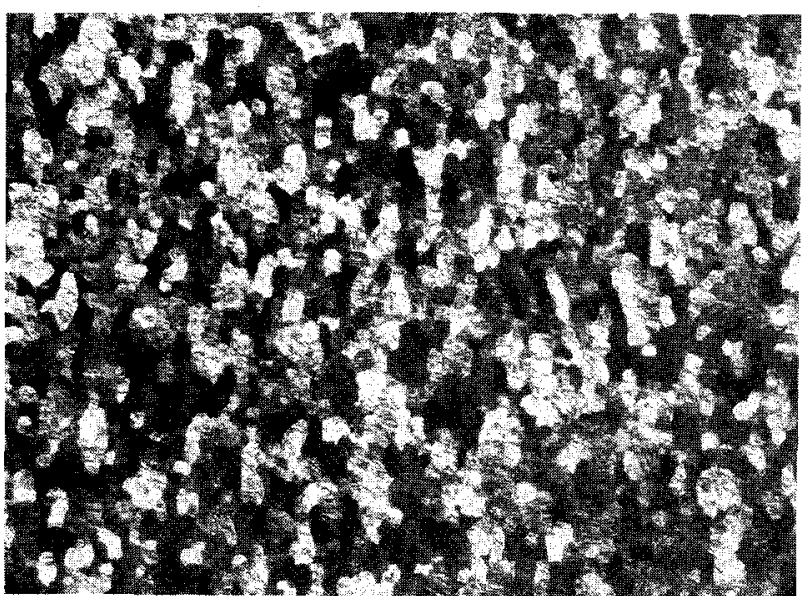

As-sputtered

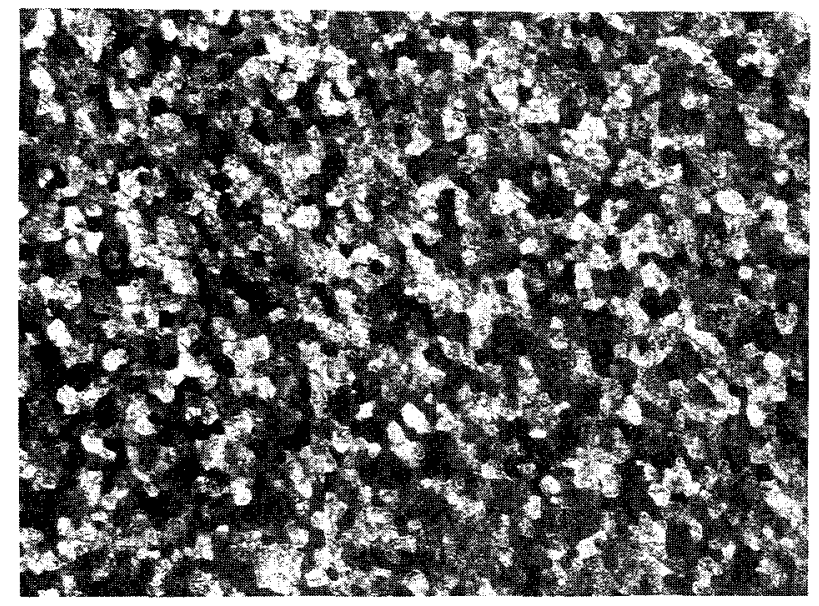

$550^{\circ} \mathrm{C}$

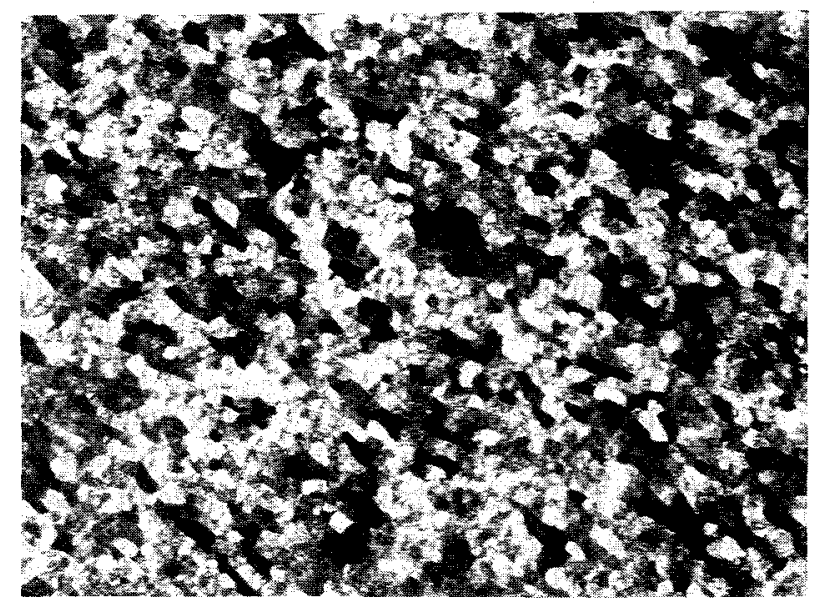

$650^{\circ} \mathrm{C}$

(b)

Fig 2. TEM micrographs of $\mathrm{Co}_{81} \mathrm{Cr}_{19}$ films (a) and $\mathrm{Co}_{81.4} \mathrm{Cr}_{16.6} \mathrm{Ta}_{2}$ films (b) before and after $550^{\circ} \mathrm{C}$ and $650^{\circ} \mathrm{C}$ anneal $\left(2.5 \mu \mathrm{m}, \mathrm{T}_{\mathrm{sub}}=100^{\circ} \mathrm{C}\right)$ 
perpendicular coercivity $\left(\mathrm{H}_{\perp}\right)$ of the two different composition films with annealing was very similar to the change of Ms. In the specimens of both compositions, when annealing temperature exceeds $500^{\circ}$ $\mathrm{C}$, Ms and $\mathrm{H}_{\perp}$ decrease very rapidly. The ternary films deposited on water cooled substrate, however, did not show any change of Ms during the same heat treatments. The drops of $\mathrm{Ms}$ and $\mathrm{H}_{\perp}$ are interpreted as being associated with redistribution of the solute atoms segregated during depositing process at a high substrate temperature. The $\mathrm{CoCr}$ film showed the increase of $\mathrm{Ms}$ after $1 \mathrm{hr}$ annealing at $150^{\circ} \mathrm{C}$ but the coercivity did not change. In the previous works ${ }^{3-5}$, this type of Ms increase was not reported. The cause of this increase is not clearly understood.

Extensive structural analyses were done for both composition specimens before and after annealing by TEM and XRD. There were no appreciable grain growth and no second phase formation up to $550^{\circ} \mathrm{C}$ and $650^{\circ} \mathrm{C}$ annealing as shown in Fig. 2.

The in-plane micrographs were taken from midsection of $2.5 \mu \mathrm{m}$ thick films. Average grain sizes of the all specimens were about $700 \AA$ while those of the assputtered films of $0.2 \mu \mathrm{m}$ thickness were in the range of $200 \sim 300 \AA$. The grain size difference is originating from conical growth characteristics of the films. The larger grain size in the $2.5 \mu \mathrm{m}$ thickness films seems also to be associated with the lower coercivity.

$\mathrm{X}$-ray diffraction showed about 1 degree increase in a half width of the rocking curve of the $c$ plane diffraction peak in both binary and ternary films after the anneal at $550^{\circ} \mathrm{C} . \Delta \Theta_{50}$ of $(0002)$ plane diffraction increased linearly with increasing annealing temperature up to $550^{\circ} \mathrm{C}$ in both composition specimens. After annealing the as-sputtered films at $550^{\circ} \mathrm{C}, \Delta \Theta_{50}$ increased from $5^{\circ}$ to $6^{\circ}$ in $\mathrm{CoCr}$ films and from $4^{\circ}$ to $5^{\circ}$ in CoCrTa films.

The fact that there is no grain growth and no appreciable c-axis alignments change during the anneal indicates the coercivity change by annealing should be associated with the solute atom redistribution.

In the earlier work $^{1}$, we have reported higher coercivity behaviour in CoCrTa system is not associated with segregation of $\mathrm{Ta}$ itself but associated with promotion of enhanced segregation of $\mathrm{Cr}$ due to the existence of $\mathrm{Ta}$ in the Co matrix. This prediction was later confirmed in a $\mathrm{CoCrTa}$ longitudinal medium by EDX studies in FE-TEM where Ta content was uniform within grains and $\mathrm{Cr}$ content was higher in grain boundary region ${ }^{6}$. To study the redistribution behaviour of the segregated solutes, we have measured the change of magnetic properties of $\mathrm{CoCr}$ and $\mathrm{CoCrTa}$ films at room temperature by varying annealing time at $550^{\circ} \mathrm{C}$. Fig. 3,4 and 5 show respectively the change of Ms, the perpendicular coercivity and the perpendicular anisotropy $\left(\mathrm{K}_{\text {prep }}\right)$ of the $\mathrm{CoCr}$ and $\mathrm{CoCrTa}$ films deposited on $100^{\circ} \mathrm{C}$ substrates.

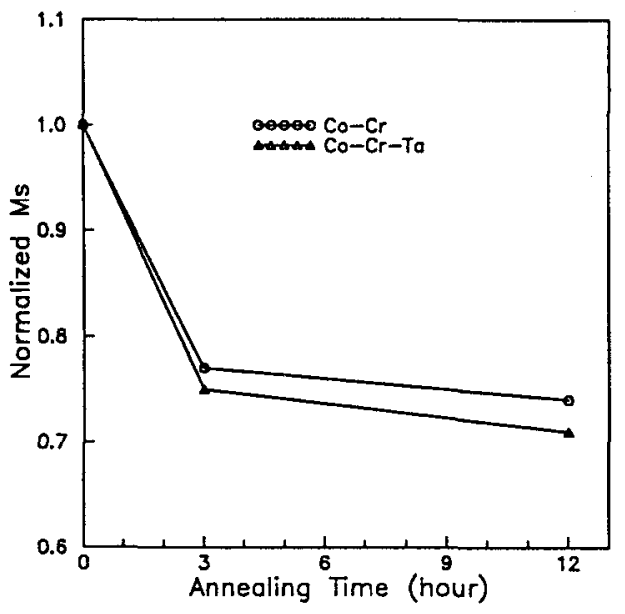

Fig 3. Variation of normalized $\mathrm{Ms}$ of $\mathrm{Co}_{81} \mathrm{Cr}_{19}$ and $\mathrm{Co}_{81.7} \mathrm{Cr}_{16.7} \mathrm{Ta}_{1.6}$ films with annealing time

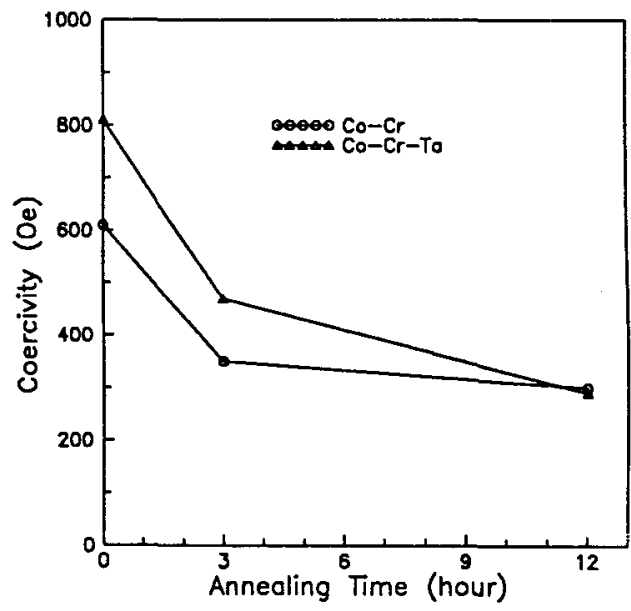

Fig 4. Variation of perpendicular coercive of $\mathrm{Co}_{81} \mathrm{Cr}_{19}$ and $\mathrm{Co}_{81.7} \mathrm{Cr}_{16.7} \mathrm{Ta}_{1.6}$ films with annealing time

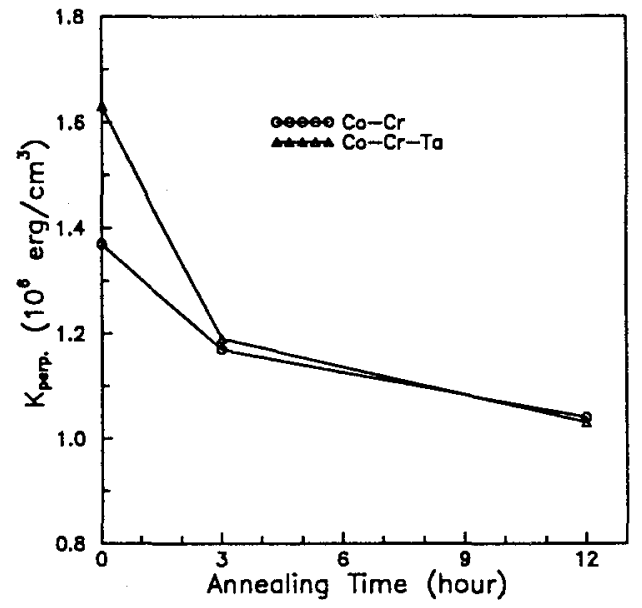

Fig 5. Variation of perpendicular anisotropy of $\mathrm{Co}_{81} \mathrm{Cr}_{19}$ and $\mathrm{Co}_{81.7} \mathrm{Cr}_{16.7} \mathrm{Ta}_{1.6}$ films with annealing time 
It is very interesting to observe that the hard magnetic properties drop more rapidly for the early stage of annealing and this trend is stronger in the ternary system. This may indicate either higher concentration of segregated solute atoms are existing in the CoCrTa system than the CoCr binary system in the as-sputtered films or the diffusivity of the solute atoms in CoCrTa films is higher than that in $\mathrm{CoCr}$ films due to the addition of larger $\mathrm{Ta}$ atoms.

Both the higher concentration gradient and the higher diffusivity will bring about more rapid drop of the coercivity and $K_{\text {perp }}$ during the anneal. Considering the amount of defects existing in as-sputtered films and the above-mentioned higher coercivity of the as-sputtered CoCrTa films, it is concluded that the concentration of the solute atoms in the segregation region is higher in the ternary films than in the binary films. After the 12 $\mathrm{hrs}$ anneal, $\mathrm{Ms}$, the perpendicular coercivity and $\mathrm{K}_{\text {prep }}$ in both films become the same value. This suggests the same kind of solute atoms is redistributing during the anneal and less than 2 at\% Ta existing in grains as solid solution is not effective in decreasing either the perpendicular coercivity or $\mathrm{K}_{\text {prep }}$.

To analyse which atomic species are redistributing during the anneal, the change of lattice spacing in both films after annealing at various temperatures for $1 \mathrm{hr}$ was measured as shown in Fig 6.

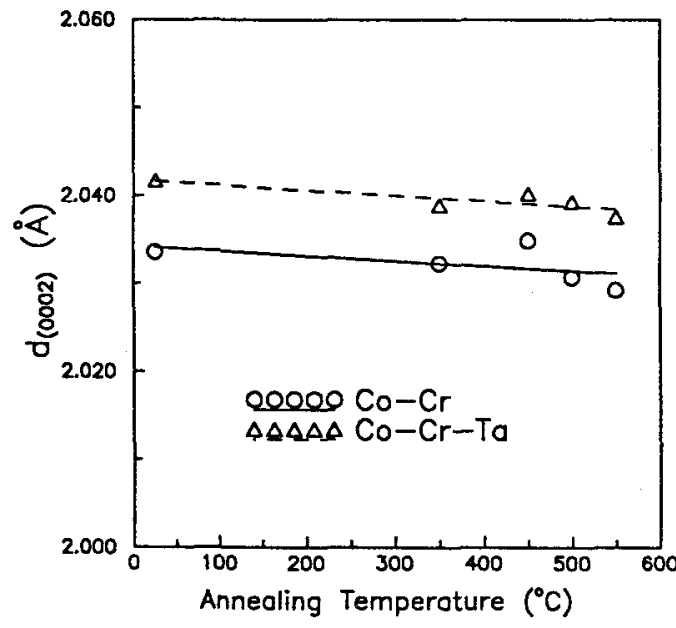

Fig 6. Variation of $\mathrm{d}$ spacing in $\mathrm{Co}_{81} \mathrm{Cr}_{19}$ and $\mathrm{Co}_{81.7} \mathrm{Cr}_{16.7} \mathrm{Ta}_{1.6}$ films with annealing time

The slopes of the decrease in the lattice spacing were the same, which strongly suggests the redistributing atomic species in both films is $\mathrm{Cr}$. If $\mathrm{Ta}$ segregated preferentially near the grain boundary should exist and be redistributed, the slope should be different in the ternary system. The decrease of $d$ spacing in both specimens may be due to either reduction of residual stress or $\mathrm{Cr}$ redistribution. However, it was found that increase of $\mathrm{Cr}$ in $\mathrm{CoCr}$ system does not change the $\mathrm{d}$ spacing as shown in Fig 7. This result suggests that the reduction in the $\mathrm{d}$ spacing in Fig 6 is due to reduction of residual stress during the annealing. From the above results, we conclude it is $\mathrm{Cr}$ which is redistributing during the anneal.

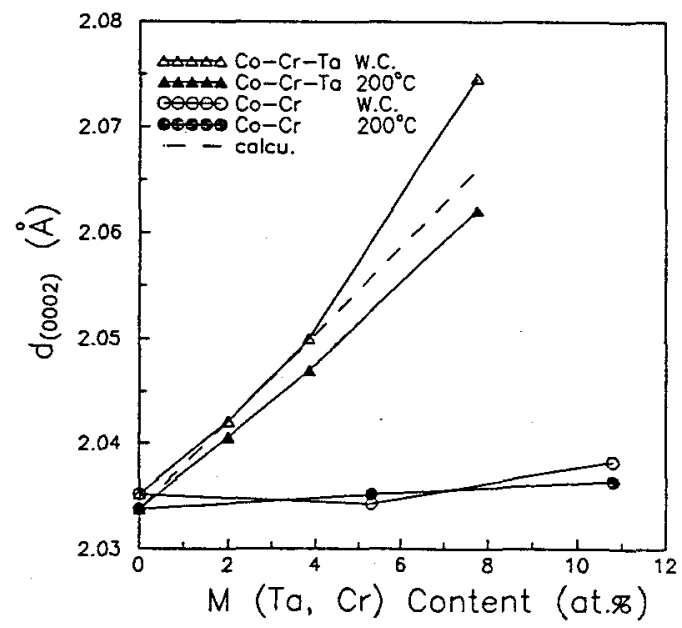

Fig 7. Variation of d spacing in $\mathrm{Co}_{81} \mathrm{Cr}_{19}$ and $\mathrm{Co}_{81.7} \mathrm{Cr}_{16.7} \mathrm{Ta}_{1.6}$ films with $\mathrm{Ta}, \mathrm{Cr}$ content

\section{ConClusion}

By analysing the results of the annealing experiments of CoCrTa films, we found that the redistributing atomic species is $\mathrm{Cr}$ rather than $\mathrm{Ta}$. Accordingly these results are supporting our earlier claim that higher coercivity in $\mathrm{CoCrTa}$ films than that in $\mathrm{CoCr}$ films is associated with the enhanced $\mathrm{Cr}$ segregation due to the existence of $\mathrm{Ta}$ in the matrix.

\section{REFERENCES}

1. C.H.Hwang, Y.S. Park, P.W.Jang and T.D.Lee: IEEE Trans. on Magnetics. 29, No 6, 3733 (1993)

2. Y. Deng, D.N.Lambeth, D.E.Laughlin: IEEE Trans. on Magnetics. 29, No 6, 3676 (1993)

3. P.W.Jang, Y.H.Kim, T.D.Lee and T.Kang: IEEE Trans. on Magnetics. 25, No 5, 4168 (1989)

4. M.Ishizuka, T.Komoda, S.Tsuchiomoto, M.Yoshikawa, S.Ishio and M.Takahashi: J. Magn.Magn. Mater. 35, 286 (1983)

5. J.E. Snyder, K.R.Mountfield and M.H.Kryder: J.Appl.Phys. 61, 3146 (1987)

6 J.Nakai, E.Kusumoto, T.Miyamoto, K.Yoshikawa and K.Itayama:

DB-11, 6th Joint MMM-Intermag Conference 